\title{
AÇÕES EDUCATIVAS E LOGÍSTICA REVERSA DE
MENTOS DESCARTADOS NA CIDADE UNIVERSITÁRIA DE \\ AÇÕES EDUCATIVAS E LOGÍSTICA REVERSA DE
MEDICAMENTOS DESCARTADOS NA CIDADE UNIVERSITÁRIA DE MACAÉ-RJ*
}

Vítor Todeschini ${ }^{1}$

ORCID: https://orcid.org/0000-0002-8749-3842

Marcos Felipe Martins Gomes ${ }^{2}$

ORCID: https://orcid.org/0000-0002-3144-3598

Júlia Ribeiro Sales ${ }^{3}$

ORCID: https://orcid.org/0000-0003-0740-027X

Thaís Marcela da Silva Pereira ${ }^{4}$

ORCID: https://orcid.org/0000-0003-0278-0365

Raissa Martins Fraga Oliveira ${ }^{5}$

ORCID: https://orcid.org/0000-0002-5994-2292

Marlon Roca dos Santos Campos 6

ORCID: https://orcid.org/0000-0001-5060-4687

Raiane Lima Santos ${ }^{7}$

ORCID: https://orcid.org/0000-0001-8868-5865

Bárbara Siqueira dos Santos 8

ORCID: https://orcid.org/0000-0003-3849-0706

Chayanne Souza Chagas 9

ORCID: https://orcid.org/0000-0002-2863-5660

Anna Karolina Mouzer S. Machado ${ }^{10}$ \footnotetext{
Universidade Federal do Rio de Janeiro.

${ }^{1}$ Universidade Federal do Rio de Janeiro.

${ }^{2}$ Universidade Federal do Rio de Janeiro.

${ }^{3}$ Universidade Federal do Rio de Janeiro.

${ }^{4}$ Universidade Federal do Rio de Janeiro.

${ }^{5}$ Universidade Federal do Rio de Janeiro.

${ }^{6}$ Universidade Federal do Rio de Janeiro.

${ }^{7}$ Universidade Federal do Rio de Janeiro.

${ }^{8}$ Universidade Federal do Rio de Janeiro.

${ }^{9}$ Universidade Federal do Rio de Janeiro.

${ }^{10}$ Universidade Federal do Rio de Janeiro.
}

* Pesquisa financiada pelo Programa Institucional de Fomento Único de Ações de Extensão - PROFAEX da 
ORCID: https://orcid.org/0000-0002-3626-2788

Juliana Givisiez Valente ${ }^{11}$

ORCID: https://orcid.org/0000-0001-9069-1754

Maria Christina dos Santos Verdam ${ }^{12}$

ORCID: https://orcid.org/0000-0002-7719-3993

Denise Oliveira Guimaraes ${ }^{13}$

ORCID: https://orcid.org/0000-0002-9883-5342

Maximiliano da Silva Sangoi ${ }^{14}$

ORCID: https://orcid.org/0000-0002-6009-4809

Thiago Barth ${ }^{15}$

ORCID: https://orcid.org/0000-0001-8534-3122

Samantha Monteiro Martins ${ }^{16}$

ORCID: https://orcid.org/0000-0003-4235-7860

Marina Cardoso Nemitz ${ }^{17}$

ORCID: https://orcid.org/0000-0001-6226-6363

Recebido em: 03/05/2021.

Publicado em: 30/09/2021.

\section{RESUMO}

Os medicamentos podem constituir risco à saúde pública e ao meio ambiente se descartados incorretamente. Neste cenário vem sendo desenvolvido o projeto "Descarte de medicamentos: diagnóstico, educação e gerenciamento em domicílios e estabelecimentos farmacêuticos do município de Macaé-RJ" cujo objetivo envolve a realização de ações educativas e o estabelecimento de procedimentos para o correto manejo desses resíduos em conformidade com as Boas Práticas de Gerenciamento dos Resíduos de Serviços de Saúde (RSS). Um sistema de logística reversa foi desenvolvido através da disponibilização de um dispositivo contentor com espaços destinados à segregação de formas farmacêuticas sólidas, semissólidas e líquidas na cidade Universitária de Macaé-RJ. Em dias pré-determinados realizou-se o recebimento dos resíduos de medicamentos, seguidos da identificação, análise e armazenamento temporário. A coleta, transporte externo, tratamento e destinação ambientalmente adequada do resíduo foram conduzidos pela empresa Transforma Gerenciamento de Resíduos, parceira do projeto e licenciada para a atividade. A caracterização dos materiais foi realizada com formulário validado observando-se $21,2 \mathrm{~kg}$ de

\footnotetext{
${ }^{11}$ Universidade Federal do Rio de Janeiro.

${ }^{12}$ Universidade Federal do Rio de Janeiro.

${ }^{13}$ Universidade Federal do Rio de Janeiro.

${ }^{14}$ Universidade Federal do Rio de Janeiro.

${ }^{15}$ Universidade Federal do Rio de Janeiro.

${ }^{16}$ Universidade Federal do Rio de Janeiro.

${ }^{17}$ Universidade Federal do Rio de Janeiro.
} 
resíduos durante o ano de 2019. Durante este mesmo ano a ação educativa "Posso jogar remédio no lixo?" foi realizada para diferentes públicos escolares do ensino médio de Macaé e região, sendo observado ótimos indicadores de satisfação junto ao público participante. Assim, além do diálogo e troca de saberes envolvendo os resíduos de medicamentos, o presente estudo estabeleceu procedimentos para o seu adequado gerenciamento, contribuindo para a saúde pública e ambiental e podendo servir como fonte de consulta para estimular e orientar novas iniciativas relacionadas ao tema.

Palavras-chave: ações educativas; logística reversa; risco à saúde; risco ao meio ambiente.

\title{
EDUCATIONAL ACTIONS AND REVERSE LOGISTICS OF MEDICINES DISCARDED IN THE UNIVERSITY CITY OF MACAÉ-RJ
}

\begin{abstract}
Medicines can be a risk to environment and public health if incorrectly discarded. In this context, the project "Discard of medicines: diagnosis, education and management in households and pharmaceutical establishments in Macaé-RJ city" has been developed. The aims involves carry out educational actions and the procedures establishiment for correct handling of medicine residues in accordance with Good Health Services Waste Management Practices. A reverse logistics system was developed through the provision of a container device in the University city of Macaé-RJ with segregation places of solid, semi-solid and liquid pharmaceutical forms. The drug waste were received on pre-determined days, followed by material identification, analysis and temporary storage. The collection, external transport, treatment and appropriate disposal was carried out by the company project partner licensed for the activity. The materials characterization was carried out using a validated form, observing a total a waste total of $21.2 \mathrm{~kg}$ during the year 2019. The educational action "Can I throw medicine in the trash?" was carried out in the same year and was apllied for different high school publics in Macaé and the region, with excellent satisfaction indicators observed among the participating public. Thus, this study was established a communication channel and knowledge exchange involving drug residues. Moreover, this study established correct procedures for drug residues management, contributing to public and environmental health and being able to serve as a source of consultation to stimulate and guide new initiatives.
\end{abstract}

Keywords: educational actions; environment risck; health risk; reverse logistic.

\section{INTRODUÇÃO}

O medicamento constitui-se, nos termos da Lei $\mathrm{n}^{\circ} 5.991$ de 17 de dezembro de 1973, como um produto farmacêutico tecnicamente obtido ou elaborado, com finalidade profilática, curativa, paliativa ou para fins de diagnóstico (BRASIL, 1973). Assim, o medicamento caracteriza-se como um instrumento essencial para a saúde pública através da manutenção, redução ou eliminação de sintomas, controle do progresso ou prevenção de doenças e enfermidades (ANVISA, 2010). 
A Política Nacional de Medicamentos, instituída na Portaria n 3.916 de 30 de outubro de 1998 e que é parte integrante da Política Nacional de Saúde, constitui um dos elementos fundamentais para a efetiva implementação de ações capazes de promover a melhoria das condições da assistência à saúde da população, tendo o importante propósito de garantir a necessária segurança, eficácia e qualidade dos medicamentos, a promoção do uso racional e o acesso da população àqueles considerados essenciais (BRASIL, 1998). Contudo, simultaneamente a todos os benefícios trazidos pela utilização dos medicamentos, estes podem constituir risco à saúde quando utilizados erroneamente, assim como um risco à saúde pública e ao meio ambiente quando descartados de forma incorreta, tornando o seu adequado estudo e gerenciamento imprescindível (MARINI, OLIVEIRA, 2015; PINTO et al., 2019).

O panorama das intoxicações por medicamentos no Brasil baseado em análise de dados do Sistema Nacional de Informações Toxicológicas (SINITOX), que é responsável por divulgar estatísticas referentes a casos de intoxicação, indicam que os medicamentos continuam sendo um dos principais agentes responsáveis por casos de intoxicações em humanos. Uma análise entre os anos de 1999 a 2017 indicou os medicamentos como responsáveis por cerca de $28,65 \%$ do número total de casos de intoxicações registrados por agente tóxico, correspondendo a um número total de 5.118,79 casos para este período (SINITOX, 2021). Vale ressaltar que as notificações ao SINITOX têm caráter voluntário e, portanto, os casos de intoxicações podem ser ainda maiores.

Além das intoxicações, o descarte incorreto de medicamentos tem potencial de expor o meio ambiente a diversas substâncias que contaminam os seres vivos por meio da água, do solo e do ar (BILA, DEZOTTI, 2003; RAMOS et al., 2017). Neste contexto, tanto os números envolvendo a contaminação ambiental por medicamentos no Brasil quanto às legislações relacionadas ainda são escassas. As consequências do descarte incorreto de medicamentos podem ser encontradas no trabalho publicado por Lima e colaboradores em 2017, onde é relatado a presença de diversas categorias de substâncias químicas, tais como medicamentos, hormônios naturais, sintéticos, e produtos industriais tanto em águas naturais, quanto nas de Estações de Tratamento de Água (ETA) e de Esgoto (ETEs). É importante destacar que na regulamentação nacional que apresenta padrões de qualidade para água potável, a Portaria $\mathrm{n}^{\circ}$ 2914 de 12 de dezembro de 2011 (BRASIL, 2011), bem como na Directiva 2008/105/EC que apresenta normas de qualidade ambiental da água na União Europeia (Council of the European Communities, 2008), os limites de medicamentos e hormônios em águas não estão 
incluídos. Porém, a União Europeia incluiu na Decisão de Implementação da Comissão 2015/495 uma lista de observação para alguns fármacos como o diclofenaco, antibióticos da família dos macrolídeos (eritromicina, claritromicina e azitromicina), e para o hormônio sintético 17-alfa-etinilestradiol (Council of the European Communities, 2015), a fim de serem monitorados nas águas superficiais para possível adição futura à lista de prioridades.

Quanto a questão da gestão de resíduos, o Brasil teve um importante marco regulatório estabelecido na Lei $\mathrm{n}^{\mathrm{o}} 12.305$ de 2 de agosto de 2010 e que instituiu a Política Nacional de Resíduos Sólidos (PNRS). Dentre os pilares fundamentais do PNRS está a logística reversa e a responsabilidade compartilhada pelo ciclo de vida dos produtos abrangendo os fabricantes, importadores, distribuidores e comerciantes, os consumidores e os titulares dos serviços públicos de limpeza urbana e de manejo de resíduos sólidos (BRASIL, 2010). É importante destacar que o PNRS não apresenta detalhamentos sobre os resíduos do serviço da saúde (RSS), estando estes conteúdos estão dispostos na Resolução RDC n ${ }^{\circ} 222$ de 28 de março de 2018 que regulamenta as Boas Práticas de Gerenciamento dos RSS da Agência Nacional de Vigilância Sanitária (BRASIL, 2018), incluindo-se os resíduos de medicamentos.

Esta resolução estabelece requisitos fundamentais para todas as etapas do manejo dos RSS, incluindo a segregação, acondicionamento, identificação, transporte interno, armazenamento temporário, armazenamento externo, coleta interna, transporte externo, destinação e disposição final ambientalmente adequada e é aplicável a todos os serviços cujas atividades estejam relacionadas com a atenção à saúde humana ou animal, sendo, portanto, compulsória por parte dos estabelecimentos farmacêuticos (BRASIL, 2018). Entretanto, a regulamentação não é aplicável aos resíduos de medicamentos provenientes de domicílios.

Neste sentido, recentemente foi publicado o Decreto $\mathrm{n}^{\mathrm{o}} 10.388$, de 5 de junho de 2020, que institui o sistema de logística reversa de medicamentos domiciliares vencidos ou em desuso. Este regulamento é específico para resíduos de medicamentos de uso humano provenientes de domicílios, tanto os medicamentos industrializados quanto os manipulados (BRASIL, 2020). Assim, fica estabelecido duas fases para implementação do sistema logístico, sendo a primeira relacionada a instituição de um grupo de acompanhamento de performance e relatórios e a segunda relacionada a habilitação de prestadores de serviço, a elaboração de plano de comunicação e a instalação de pontos fixos de recebimento de medicamentos domiciliares vencidos ou em desuso (BRASIL, 2020).

Como pode ser observado, avanços nas regulamentações envolvendo o descarte de 
medicamentos vem ocorrendo. Contudo, a implementação e consolidação das diretrizes nelas estabelecidas demandam tempo, além das práticas de conduta ainda não estarem difundidas na comunidade. Desta forma, o descarte inadequado destes resíduos permanece sendo uma dificuldade, podendo comprometer a saúde de indivíduos devido a intoxicações pela reutilização, além dos danos ao meio ambiente.

Sabe-se da responsabilidade da Universidade em se aproximar da sociedade e de suas demandas. Uma forma de interagir com a sociedade de forma a causar mudanças nas estruturas sociais, e inserir o discente na comunidade promovendo mudança em sua postura cidadã, é a extensão universitária em suas múltiplas ações (BRASIL, 2015). Desta forma, unindo o universo acadêmico farmacêutico, bem como a importância da temática e seu impacto ambiental, e a necessidade de tornar acessível o conhecimento do tema pela sociedade, e ainda entender como as questões de impacto ambiental e medicamento, e a relação do tema com a sociedade, foi criado o projeto de extensão "Descarte de medicamentos: diagnóstico, educação e gerenciamento em domicílios e estabelecimentos farmacêuticos em Macaé-RJ". O objetivo consiste na implantação de ações educativas e a troca de saberes entre a Universidade Federal do Rio de Janeiro-Campus Macaé e a comunidade de Macaé e região. Além disso, objetiva estabelecer um sistema de logística reversa de medicamentos domiciliares vencidos ou em desuso na cidade Universitária de Macaé-RJ e a caracterização desses resíduos.

\section{METODOLOGIA}

Este trabalho foi realizado de acordo com os conceitos da extensão universitária, sendo construído de forma colaborativa entre docentes e alunos do Curso de Farmácia da Universidade Federal do Rio de Janeiro-Campus Macaé (UFRJ-Macaé) e tendo como base as ações conduzidas pelo projeto intitulado "Descarte de medicamentos: diagnóstico, educação e gerenciamento em domicílios e estabelecimentos farmacêuticos do município de Macaé-RJ" durante o ano de 2019.

As pesquisas bibliográficas que embasaram teoricamente o desenvolvimento projeto e este trabalho foram realizadas em diferentes bases de dados, incluindo o portal Periódicos CAPES, assim como em agências reguladoras e plataformas governamentais de saúde e meio ambiente e usando diversos descritores tais como "Descarte de medicamentos", "Resíduos de 
serviços da saúde", "Resíduos sólidos" e "Uso racional de medicamentos".

O sistema de logística reversa foi estabelecido contemplando as diferentes etapas do manejo de resíduos de medicamentos e em conformidade com determinações regulamentares, incluindo-se as etapas de recebimento, segregação, acondicionamento, identificação, armazenamento, transporte, tratamento e disposição final. Para tal, foi desenvolvido e implantado um dispensador contentor dos resíduos (coletor) com espaços delimitados para formas farmacêuticas sólidas, líquidas e semissólidas no polo universitário da UFRJ-Macaé. Para este trabalho, o recebimento dos medicamentos vencidos ou em desuso neste coletor ocorreu durante o ano de 2019 e em dias e horários pré-determinados e devidamente divulgados à comunidade interna e externa através do uso de cartazes e redes sociais. A localização do coletor (ponto de recebimento ou coleta dos resíduos domiciliares) foi estabelecida em ambiente de grande circulação de pessoas e visibilidade no polo universitário da UFRJ-Macaé.

A qualificação e quantificação dos materiais coletados, por sua vez, foram conduzidas com o auxílio de um sistema documental desenvolvido no presente estudo. A coleta externa dos medicamentos vencidos ou em desuso armazenado no polo universitário, transporte e destinação ambientalmente correta foi conduzida pela empresa macaense Transforma Gerenciamento de Resíduos, parceira fundamental do projeto de extensão.

Considerando os objetivos do projeto de extensão foram realizadas ações educativas nas dependências do polo universitário da UFRJ em Macaé. Essas ações foram elaboradas considerando as etapas de planejamento, execução e avaliação em convergência às etapas da norma orientativa da Associação Brasileira de Normas Técnicas (ABNT) relacionada à treinamentos (ABNT, 2020). Ressalta-se que as atividades foram avaliadas pelo público-alvo para verificar a satisfação e rastreabilidade documental, além de servir de subsídio para a melhoria contínua das ações futuras. Para tal, formulário de satisfação foi elaborado e disponibilizado ao final das atividades, sendo seu preenchimento voluntário e anônimo. Por se tratar de uma pesquisa de satisfação, o formulário não necessitou aprovação prévia por Comitê de Ética em Pesquisa (BRASIL, 2016).

\section{RESULTADOS E DISCUSSÃO}

O projeto de extensão intitulado "Descarte de medicamentos: diagnóstico, educação e gerenciamento em domicílios e estabelecimentos farmacêuticos em Macaé-RJ" foi 
devidamente aprovado pelas instâncias competentes da UFRJ e vem realizando suas ações desde o segundo semestre de 2016, tendo como objetivo fundamental o estabelecimento de um canal de diálogo entre a instituição e sociedade. Dessa forma, o projeto busca estabelecer um sistema de logística reversa de medicamentos vencidos ou em desuso, apresentando uma importante solução ao descarte desses materiais para a população, bem como difundir o conhecimento envolvendo o descarte de medicamentos, contribuindo para a melhoria da qualidade de vida da população e conservação do meio ambiente.

O projeto foi elaborado tendo como princípio norteador as diretrizes da extensão universitária. Desta forma, a participação da equipe, em especial de discentes ocorreu ao longo do projeto incluindo a elaboração e aplicação das atividades educativas junto aos públicos-alvo e análise crítica das ações e a orientação, recebimento, análise e armazenamento temporário dos resíduos de medicamentos, além da participação em eventos científicos e

apresentação de trabalhos. É importante destacar que, com o objetivo de definir uma identidade do projeto de extensão e potencializar a efetividade das atividades educativas junto aos públicos-alvo, a equipe executora criou através da união das palavras "remédio e lixo", o personagem denominado Remelixo®, Figura 1. A marca (imagem e nomenclatura) encontrase registrada junto ao Instituto Nacional da Propriedade Industrial (INPI) para garantia da propriedade e do uso exclusivo.

Reuniões periódicas para discussões, análise crítica e definição de estratégias e planejamento das atividades, como oficinas propostas em eventos de extensão no polo universitário, escala de horários para presença efetiva próximo ao coletor, seja para receber de maneira correta ou orientar sobre descarte e apresentação de trabalhos em eventos, foram atividades constantes, e com participação democrática de todos os membros do projeto. Cabe destacar o uso constante de aplicativos de mensagens, correspondência eletrônica e ambientes virtuais para troca de saberes, discussões técnico-científicas e armazenamento e gestão documental.

\subsection{Sistema de logística reversa de resíduos de medicamentos}




\subsubsection{Desenvolvimento e implantação de dispensador contentor de medicamentos}

Conforme descrito no Decreto $\mathrm{n}^{\mathrm{o}} 10.388$ de 5 de junho de 2020 , que institui o sistema de logística reversa de medicamentos domiciliares vencidos ou em desuso, fica estabelecido a instalação de pontos fixos de recebimento desses resíduos em estabelecimentos farmacêuticos considerando um cronograma e número de habitantes do município (BRASIL, 2020). Contudo, a legislação não define a estrutura, formato e dimensões do dispositivo contentor. Uma orientação de estrutura do coletor foi introduzida na norma da Associação Brasileira de Normas Técnicas - ABNT NBR 16457 de 2016. As orientações indicam que o coletor deve estar identificado, conter instruções quanto aos tipos de resíduos proibidos e permitidos a serem descartados, possuir especificação de sua capacidade em volume e massa, ser rígido e resistente à puncturas, rupturas e vazamentos, ter um mecanismo de antirretorno, ter a boca de coleta com altura entre $0,90 \mathrm{~m}$ e 1,20 m em relação ao solo, possibilitar o amortecimento da queda dos medicamentos durante o descarte, evitar o risco ocupacional e garantir o manejo apropriado (ABNT, 2016).

A estrutura física interna e externa do dispositivo contentor utilizado para recebimento dos resíduos de medicamentos no presente trabalho foi planejado e produzido pela equipe executora do projeto te extensão utilizando como base as referências normativas e as particularidades do manejo, inclusão, retirada e acesso aos materiais e transporte no polo universitário. Para tal definiu-se o material de aglomerado de madeiras e os espaços delimitados para segregação de formas farmacêuticas sólidas, líquidas e semissólidas, além de porta posterior com fechadura para manuseio do material plástico que acondiciona os resíduos e rodas de transporte. As imagens, cores e escritas foram planejadas pela equipe executora, sendo impresso e adesivado por empresa contratada. As dimensões, formato e personalização com material gráfico orientativo estão apresentados na Figura 1. 
Figura 1 - Dispositivo contentor de medicamentos vencidos ou em desuso e imagem e nomenclatura alusiva ao projeto de extensão. Onde: $\mathrm{S}=$ resíduo sólido; $\mathrm{SS}$ = resíduo semissólido; $\mathrm{L}$ = resíduo líquido.
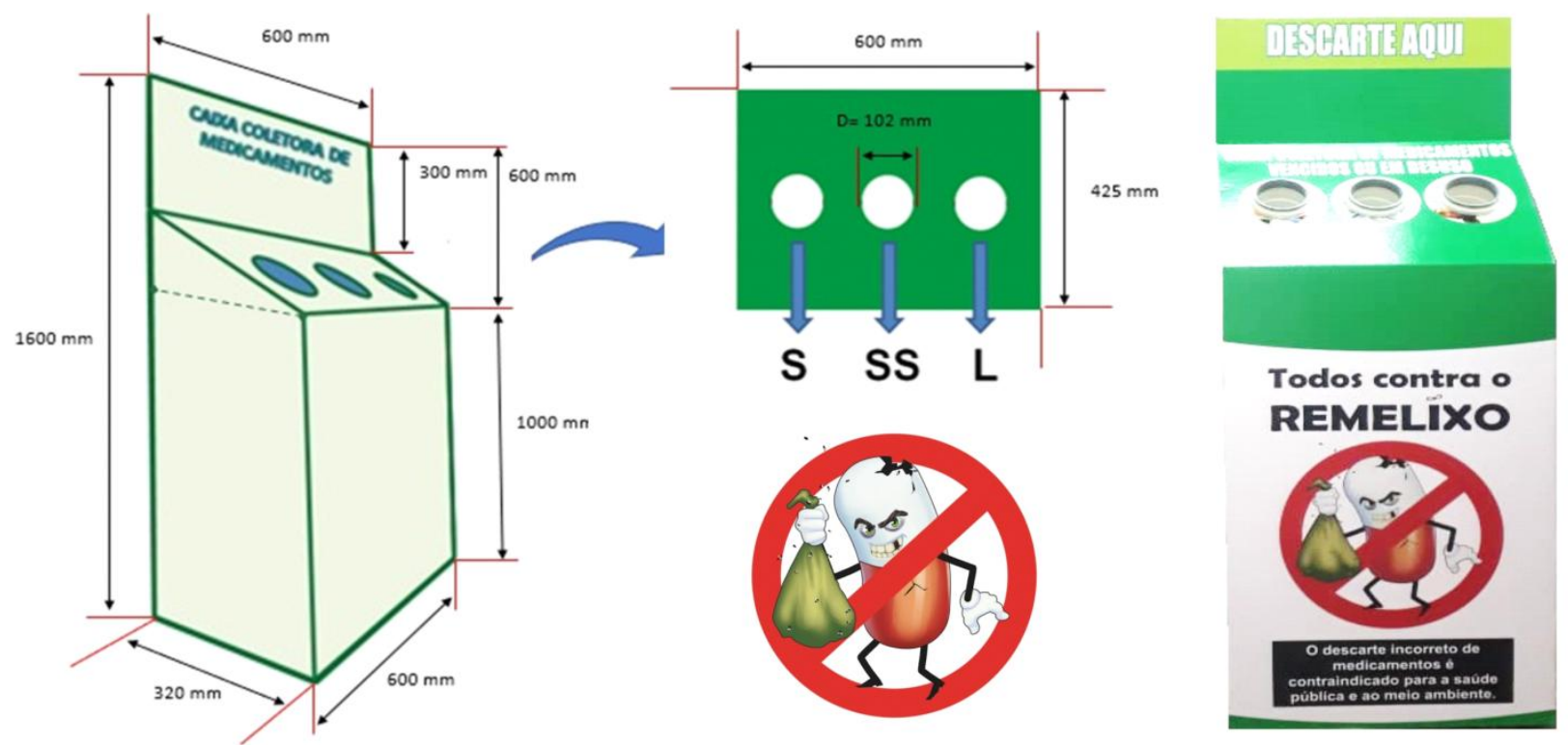

Fonte: Elaborado pelos autores.

É importante destacar que o dispositivo foi desenvolvido e vem sendo disponibilizado no polo universitário desde agosto de 2016, tendo sido recebido e destinado de forma ambientalmente correta um total de $120,1 \mathrm{Kg}$ de resíduos de medicamentos durante até o presente momento. Contudo, devido a experiência adquirida e aprimoramento contínuo em relação as práticas de gestão, a estratégia para recebimento dos resíduos de medicamentos sofreu ajustes ao longo do tempo, mais especificamente em relação ao calendário de recebimento, análise estatística, coleta externa e destinação dos resíduos equacionada com as atividades curriculares dos alunos envolvidos e empresa parceira. Assim, a estratégia utilizada foi o recebimento orientado dos resíduos, ou seja, alunos participantes do projeto de extensão ficaram disponíveis juntamente ao dispositivo contentor nos dias e horários divulgados antecipadamente em rede social e cartazes, dialogando e auxiliando o público conforme necessário. Diversas foram as orientações ao público envolvido com o descarte dos materiais, incluindo informações sobre os tipos de formas farmacêuticas e orifícios de depósito, explicações sobre armazenamento e prazo de validade e as abordagens para adequada gestão dos resíduos de medicamentos. Além disso, foi sempre salientado a necessidade do descarte dos produtos em suas embalagens primárias e/ou secundárias, permitindo a caracterização correta dos materiais e garantindo a integridade dos envolvidos e do próprio sistema de coleta, 
sem possibilidades de recebimento de materiais perfurocortantes ou afins. Em virtude da atual pandemia da COVID-19, as atividades do projeto foram momentaneamente suspensas.

\subsubsection{Manejo dos resíduos de medicamentos}

Conforme mencionado anteriormente, a Resolução RDC n 222 de 28 de março de 2018 da ANVISA regulamenta as Boas Práticas de Gerenciamento dos resíduos de serviços de saúde. Nesta resolução estão classificados os RSS em grupos de risco A (biológicos), B (químicos, incluem-se os medicamentos), C (radioativos), D (equivalentes aos domiciliares) e E (perfurocortantes), determinando também a importância do estabelecimento de um Plano de Gerenciamento dos RSS por parte dos estabelecimentos geradores (BRASIL, 2018).

Neste sentido, o gerenciamento dos resíduos relacionados ao projeto de extensão vem sendo conduzido através do planejamento e execução das etapas de manejo desses resíduos em conformidade o estabelecido na legislação vigente, incluindo-se a segregação, acondicionamento, identificação, transporte interno, coleta externa, armazenamento externo, transporte externo, destinação e disposição final. Para tal, os materiais previamente segregados em sacos individualizados pelo próprio dispositivo contentor foram acondicionados em recipientes plásticos invioláveis, sendo devidamente identificados e mantidos em armário exclusivo com chave (presente em laboratório com acesso limitado) para caracterização e posterior coleta e transporte externo.

O transporte interno foi realizado pela equipe executora do projeto do ponto de recebimento no polo universitário até o local de armazenamento temporário através do translado do dispositivo contentor que possui sistema de rodas para auxiliar a movimentação. A coleta e transporte externo dos materiais, por sua vez, foram realizadas pela empresa parceira devidamente licenciada para a atividade e utilizando veículo apropriado. Estes resíduos foram conduzidos até as dependências da referida empresa para armazenamento externo, tratamento e destinação final ambientalmente adequada. A Figura 2 apresenta imagens ilustrativas de algumas etapas relacionadas ao manejo dos resíduos de medicamentos do projeto de extensão. A parceria com setores diversos da sociedade fortalece o eixo extensionista do projeto, reforçando a interação dialógica. Além de ter a sociedade em parceria com a universidade, refletindo sobre problemas ambientais, permite aos discentes que entendam processos estudados em sala de aula, de forma prática, se inserindo na comunidade 
de forma real, o que gera impacto na formação do estudante, uma importante diretriz da extensão universitária. Destaca-se que uma visita técnica foi realizada pelos integrantes do projeto para conhecer a destinação final dos resíduos e compreender sobre os procedimentos estabelecidos. Na ocasião a equipe foi recepcionada pelos colaboradores da empresa Transforma Gerenciamento de Resíduos, sendo apresentadas todas as etapas de transformação e/ou destinação dos resíduos de acordo com as legislações adequadas.

Figura 2 - Imagens ilustrativas de etapas do manejo dos resíduos de medicamentos. Descarte de medicamentos no dispositivo contentor (a); Armário para guarda temporária dos resíduos após avaliação do perfil dos produtos descartados (b); Conferência dos documentos para coleta e transporte dos resíduos pela empresa parceira (c); Organização dos resíduos em veículo para realização do transporte externo (d); Registro dos docentes do projeto e colaboradores da empresa parceira durante a coleta dos resíduos (e); Visita técnica dos integrantes do projeto na empresa parceira (f). Imagens anteriores a pandemia da COVID-19.

a)

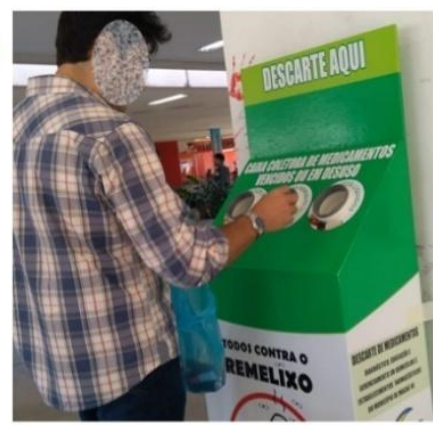

b)

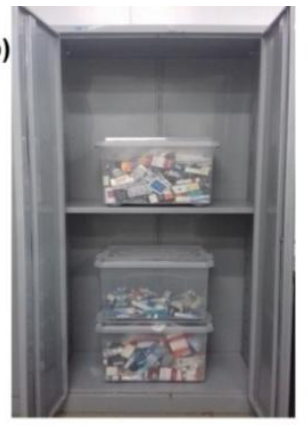

e)

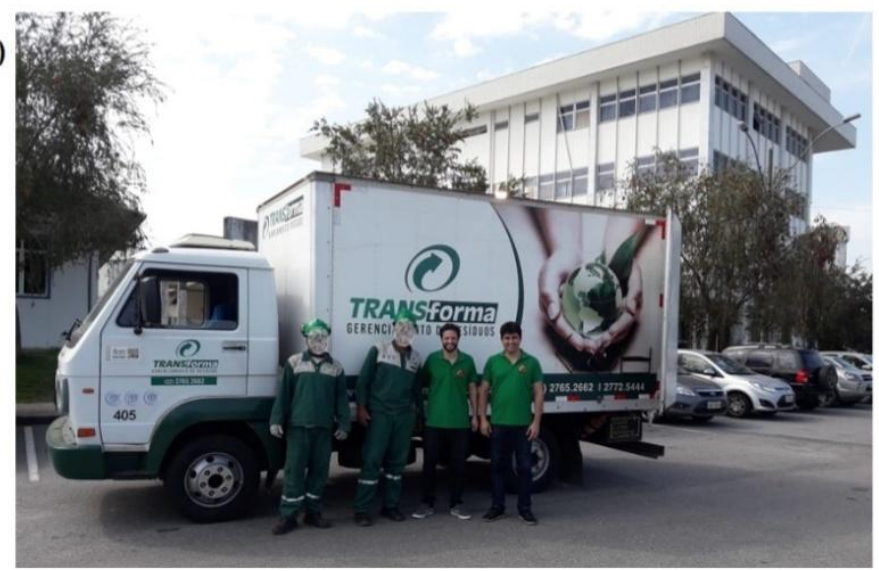

c)

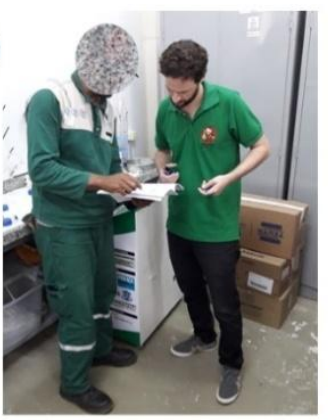

d)

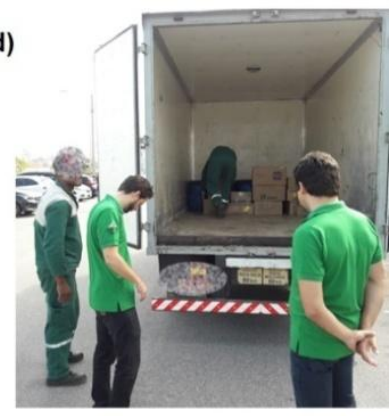

f)

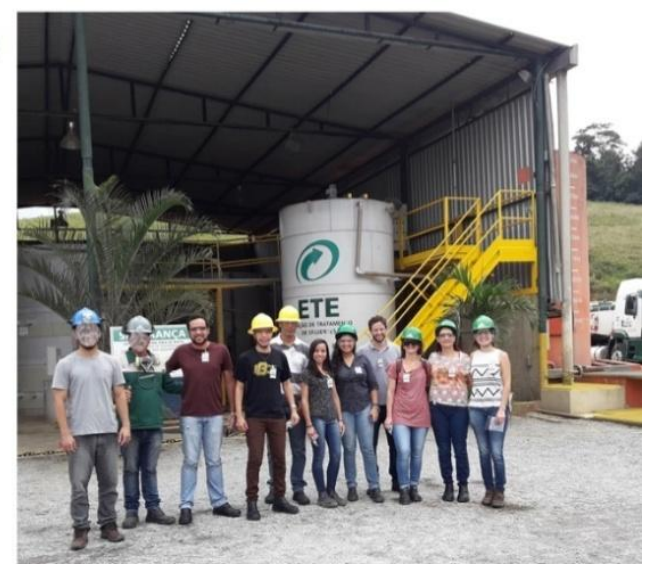

Fonte: Elaborado pelos autores.

Destaca-se, ainda, que os procedimentos foram conduzidos em conformidade com a legislação vigente e com a devida segurança dos participantes. Além disso, foram gerados os Manifestos de Transporte de Resíduos (MTR) para registro das informações dos resíduos desde a fonte geradora até a sua destinação final em conformidade com o estabelecido pelos 
órgãos ambientais. Além de compulsórios na gestão de resíduos, esses documentos são parte integrante do sistema documental do projeto de extensão, sendo informados a forma física dos materiais (sólido, líquido ou semissólido), a classificação de resíduos usada pelo Instituto Brasileiro do Meio Ambiente e dos Recursos Naturais Renováveis (IBAMA) e o peso (Kg) do material.

\subsubsection{Caracterização dos resíduos de medicamentos}

Outro importante objetivo do trabalho esteve relacionado ao estudo dos resíduos de medicamentos descartados no polo universitário de Macaé-RJ. A análise qualitativa e quantitativa dos materiais teve como base o desenvolvimento de um formulário físico e eletrônico que compõe a estrutura documental do projeto de extensão e que garante a rastreabilidade dessas atividades. Assim, após armazenamento temporário os resíduos recebidos, estes foram classificados segundo a forma farmacêutica (sólida, semissólida, líquida e subdivisões), o grupo anatômico principal segundo a classificação internacional ATC (Anatomical Therapeutic Chemical) da Organização Mundial da Saúde (OMS), a condição de descarte (produto com prazo de validade vigente ou expirado), além do enquadramento segundo as legislações vigentes para medicamentos controlados e antimicrobianos (BRASIL, 1998, BRASIL, 2011; WORLD HEALTH ORGANIZATION, 2021).

Os dados apresentados no presente trabalho foram obtidos durante o ano de 2019, sendo a geração das planilhas e análise dos resíduos realizada mensalmente pela equipe executora. Assim, foram recebidos $21,2 \mathrm{~kg}$ de resíduos de medicamentos no interstício, sendo 27,1\% de sólidos $(5,75 \mathrm{~kg}), 56,1 \%$ de líquidos (11,9 kg) e 16,7\% de semissólidos $(3,55 \mathrm{~kg})$. Mesmo com um peso total inferior às formas farmacêuticas líquidas, as formas sólidas representaram $81,9 \%$ do quantitativo de unidades analisadas. Além disso, observou-se que 85,5\% das formulações sólidas analisadas eram compostas por comprimidos e cápsulas, 68,9\% das formulações líquidas eram compostas por soluções, suspensão e xaropes e 93,5\% das formulações semissólidas compostas por cremes, géis e pomadas. Ressalta-se que foi considerado uma unidade a embalagem primária contendo qualquer quantidade de medicamento. Comparando os resultados obtidos com outros trabalhos de monitoramento de 
resíduos de medicamentos pode-se observar que a grande maioria dos descartes correspondem a medicamentos na forma sólida, apresentando percentagens equivalentes a mais de $50 \%$ (ROCHA, 2009) ou próximo de 90\% (NUNES et al., 2018; SOARES et al., 2017; BALDONI et al., 2015), sendo comprimidos e cápsulas as formas farmacêuticas mais comumente descartadas (ROCHA, 2009; BALDONI et al. 2015).

Outro dado importante está relacionado a condição de descarte e ao prazo de validade dos produtos. A análise dos materiais permitiu observar que 68,8\% dos medicamentos apresentavam o prazo de validade expirado e que $24,4 \%$ dos medicamentos foram descartados ainda com o prazo de validade vigente, ou seja, o descarte é proveniente do desuso dos mesmos. Observou-se, ainda, que 6,8\% dos produtos estavam com a data de validade ilegível.

Quanto a classificação de controle especial da ANVISA, por sua vez, os medicamentos isentos de controle especial (medicamentos de venda livre) totalizaram 78,7\% dos produtos descartados, enquanto os medicamentos controlados representaram 11,3\% e os medicamentos ilegíveis $10 \%$ do total. Já em relação à classificação ATC, os grupos descartados do montante geral de medicamentos e que merecem destaque são aqueles produtos relacionados ao Aparelho digestivo e metabolismo (13,7\%), do Sistema cardiovascular (6,7\%), Agentes anti-infecciosos para uso sistêmico (9,5\%), Sistema Nervoso $(9,1 \%)$ e os produtos dermatológicos $(10,1 \%)$, sendo estes últimos essencialmente representado por formulações semissólidas.

Apesar dos dados comparativos relacionados ao mecanismo de ação dos medicamentos descartados ainda serem escassos, alguns trabalhos apontam que os agentes anti-infecciosos apresentam percentagens variando entre 9,2\% - 16,7\% (SOARES et al., 2017; MENEZES et al., 2015; ROCHA, 2009; MISTURINI, 2015) o que corrobora com os resultados apresentados no presente estudo. Para os medicamentos que atuam no sistema cardiovascular foram encontrados valores de percentagens com maior variação que para antiinfecciosos: 32,5\% (SOARES et al., 2017); 3,55\% (MENEZES et al., 2015); 18,5\% (ALMEIDA, 2017) e 7,8\% (MISTURINI, 2015), sendo este último um resultado mais similar ao descrito neste trabalho. Considerando os medicamentos que atuam no sistema nervoso pode-se sugerir que os resultados aqui apresentados corroboram com os valores de 3,15\% 23,7\% encontrados na literatura (MENEZES et al., 2015; MISTURINI et al., 2015; SOARES et al., 2017; ALMEIDA et al., 2017). Apenas um trabalho foi encontrado com valores para 
medicamentos que atuam no sistema digestivo e metabolismo e o resultado de 43,9\% (ALMEIDA, 2017) é consideravelmente superior ao 13,7\% evidenciados no presente estudo.

\subsection{Ações educativas nas dependências do polo universitário}

Paralelamente à condução do sistema de gerenciamento dos resíduos de medicamentos, a equipe executora do projeto de extensão vem constantemente criando estratégias de troca de informações com a comunidade sobre a temática. Dentre essas ações estão incluídos o desenvolvimento e distribuição de material informativo, a disponibilização de conteúdos em

mídia social e, em especial, a realização de oficinas educacionais para diferentes públicos do município de Macaé e região, visando o diálogo, troca de saberes e sensibilização sobre a assertividade de conduta.

\subsubsection{Planejamento}

A equipe executora definiu a nomenclatura "Posso jogar remédio no lixo?" como adequada e ilustrativa para a ação junto ao público-alvo. Além disso, foi determinado os conteúdos que deveriam ser abordadas durante as oficinas, incluindo-se a contextualização do curso de farmácia, apresentação sobre formas farmacêuticas sólidas, líquidas e semissólidas, explicações sobre controle de qualidade, posologia, armazenamento nas residências, tipos de embalagens e prazo de validade de medicamentos e, em especial, o impacto do descarte incorreto de medicamentos no meio ambiente e os procedimentos para adequada gestão dos mesmos. Destaque também foi dada ao uso racional de medicamentos e aos riscos da automedicação, da interrupção e da troca da medicação prescrita, bem como quanto à necessidade da receita médica e as diferenças em relação aos medicamentos de venda livre, sempre enfatizando a importância na busca por profissionais capacitados para orientação precisa. Definiu-se, também, a necessidade da realização de dinâmicas em grupo com problematizações, fazendo uso de recursos audiovisuais e materiais impressos para preenchimento, fichas lúdicas com imagens, uso de embalagens e bulas de medicamento, entre outros.

A ação foi planejada para ser disponibilizada em diferentes oportunidades e eventos, possuindo inicialmente como público-alvo os alunos de ensino médio do município de Macaé e região. Devido às limitações de espaço no laboratório de Controle de Qualidade Físico- 
Químico do Curso de Farmácia da UFRJ-Campus Macaé definiu-se o número máximo de 15 alunos por atividade e perfazendo uma carga horária de 1 hora.

\subsubsection{Execução}

As ações apresentadas no presente trabalho ocorreram durante a realização de eventos protagonizados na cidade Universitária de Macaé-RJ, incluindo a "Semana Nacional de Ciência e tecnologia" e o "Conhecendo a UFRJ" realizados durante o ano de 2019, totalizando um total de 06 turmas e 90 participantes. Destaca-se que em todas as situações o convite para a participação e o encaminhamento do público participante até o laboratório foi realizado pela equipe responsável pela organização do evento. Uma vez recepcionados, o grupo de alunos participantes foi acomodado no espaço laboratorial. A atividade iniciou com a apresentação em projetor e diálogo sobre o curso de farmácia, os tipos de formas farmacêuticas, detalhamentos sobre a posologia, a necessidade da avaliação do prazo de validade dos medicamentos, armazenamento dos medicamentos e sobre o que fazer com medicamentos que não são mais utilizados nas residências.

Paralelamente, foram distribuídos kits contendo embalagem secundária de medicamento e formulário para preenchimento, além de fichas simulando os diferentes tipos de formas farmacêuticas. Os participantes foram então convidados a realizar uma dinâmica simulando o descarte dos medicamentos, adicionando as fichas em recipientes identificados por: "lixo comum, pia, vaso sanitário, guardar em casa, doação, outros". Ao fim da dinâmica, foram discutidos os riscos do descarte de medicamentos nos diferentes ambientes, fazendo uso de vídeos e inclusive testes práticos para avaliação de $\mathrm{pH}$ de água na presença e ausência de resíduos de medicamentos.

Para finalizar a atividade, foi apresentado o dispositivo contentor para descarte consciente desses resíduos e folder informativo pertencente ao projeto de extensão. A Figura 3 apresenta algumas imagens das oficinas realizadas nas dependências do polo universitário. 
Figura 3 - Imagens ilustrativas da ação educativa intitulada "Posso jogar remédio no lixo?" realizadas no polo universitário. Apresentação da atividade pelo discente do projeto (a); Diálogo entre discentes do projeto e estudantes do ensino médio sobre as problemáticas envolvidas pelo descarte incorreto de medicamentos (b); Apresentação do sistema de coleta de resíduos de medicamentos criado pelo projeto (c); Participação dos estudantes do ensino médio durante uma dinâmica realizada na atividade (d); Registro dos integrantes do projeto (discentes e docentes) e estudantes do ensino médio (e). Imagens anteriores a pandemia da COVID-19.

a)

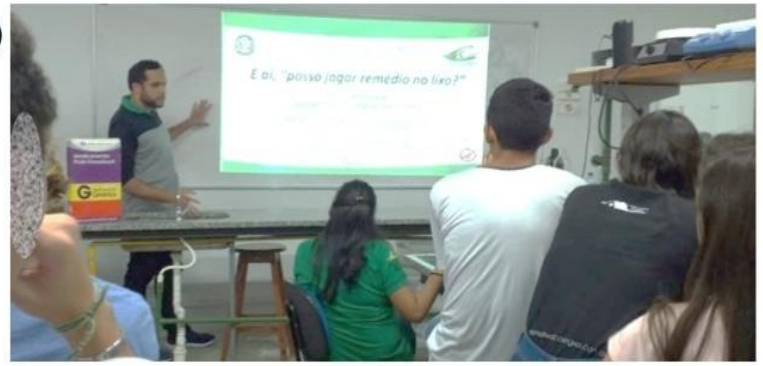

c)

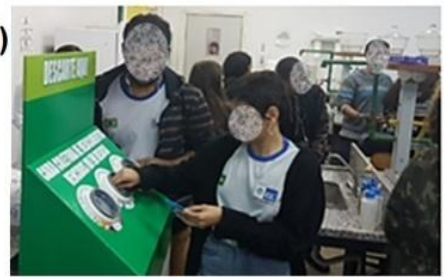

Fonte: Elaborado pelos autores.
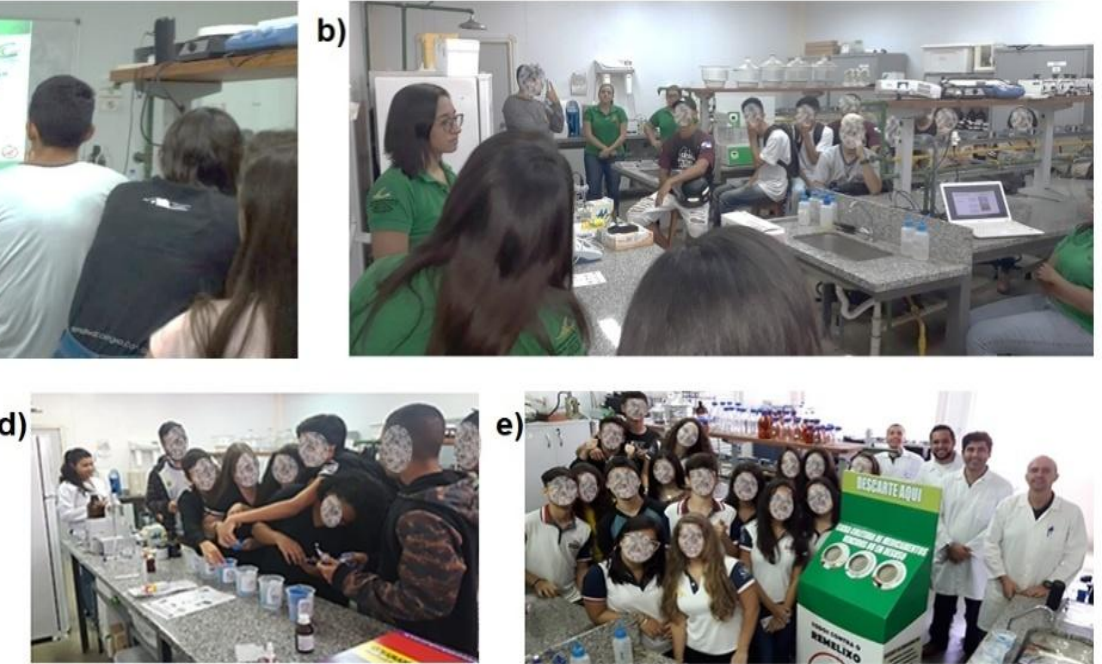

\subsubsection{Avaliação}

Visando o aprimoramento contínuo da ação e validação da atividade educativa, foi desenvolvido e aplicado, em algumas turmas, formulários de avaliação para compreender a satisfação do público externo participante, assim como propor melhorias ao andamento das atividades. Assim, uma vez finalizadas as atividades educativas foram distribuídos dois formulários, um para os alunos participantes e outro para o profissional responsável pelos alunos participantes da atividade, sendo ambos de preenchimento anônimo e voluntário. $\mathrm{O}$ público-alvo que respondeu aos formulários representou um total de 34 alunos e três professores.

O formulário para os alunos foi formado por duas perguntas : "Você gostou da atividade?" e "Você pretende repassar as informações adquiridas à sua família e amigos?", além de espaços para sugestões, críticas e elogios e campos para marcar a opção "SIM ou $N \tilde{A} O$ ” como respostas das perguntas. Já o formulário para os profissionais teve as opções de indicação de Péssimo, Ruim, Regular, Bom, Ótimo e Não se aplica para os seguintes itens de avaliação: Relevância do assunto abordado (Acredita que o tema seja importante para o dia a 
dia dos alunos?); Organização da equipe executora; Linguagem da comunicação (A linguagem estava clara? A mensagem proposta ocorreu tranquilamente?); Recursos audiovisuais (Os recursos audiovisuais utilizados durante a atividade foram satisfatórios?); Dinâmica da atividade (A dinâmica utilizada foi produtiva e adequada para a faixa etária dos alunos?); Postura da equipe executora; Tempo usado para o decorrer da atividade; Infraestrutura do local onde a atividade foi executada; Interesse dos alunos pela a atividade. A Figura 4 apresenta os resultados obtidos através dos formulários de avaliação externa (total de 34 alunos e três professores respondentes).

Figura 4 - Avaliações externas das atividades educativas nas dependências do polo universitário pelos alunos participantes (a) e pelos professores responsáveis pelos alunos (b).

a)

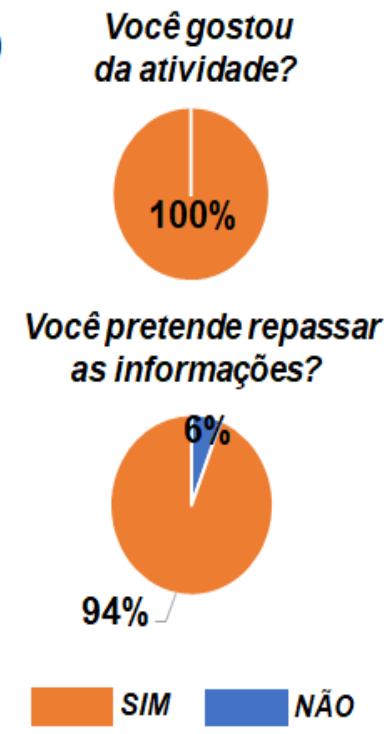

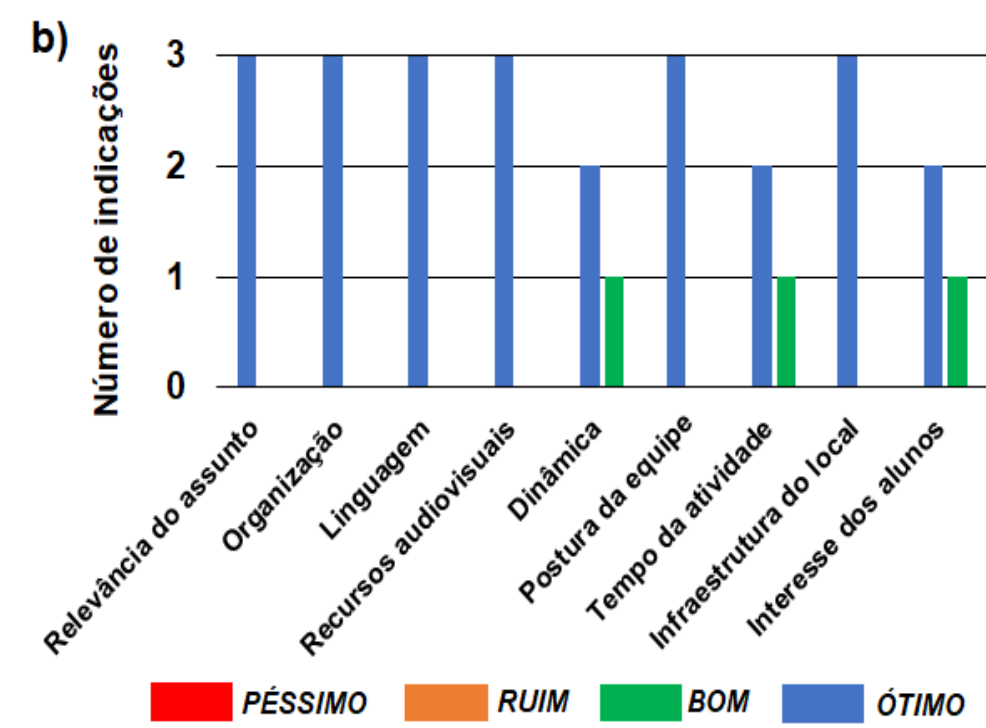

Fonte: Elaborado pelos autores.

Como pode ser observado, as respostas do formulário de avaliação apresentaram resultados satisfatórios, sendo um importante indicativo do desempenho da ação. Cabe ressaltar, ainda, que em todas as oportunidades a atividade teve ótima receptividade por parte dos estudantes e professores, sendo evidenciada através dos diversos questionamentos durante as atividades e conversas ao final da ação. Considera-se, portanto, que as atividades atingiram de forma exitosa os objetivos almejados e pode ser replicada com as devidas adaptações para outros públicos e em outras localidades. 


\section{CONCLUSÕES}

Os medicamentos são agentes terapêuticos essenciais no sistema de saúde. Contudo, quando estes produtos não são mais utilizados ou atingem seu prazo de validade, podem tornar-se prejudiciais à saúde pública e ao meio ambiente caso descartados incorretamente. Neste contexto, o presente trabalho teve como objetivo apresentar atividades e dados obtidos em 2019 pelo projeto de extensão intitulado "Descarte de medicamentos: diagnóstico, educação e gerenciamento em domicílios e estabelecimentos farmacêuticos do município de Macaé-RJ" e que envolvem o estabelecimento de um sistema de logística reversa de medicamentos domiciliares vencidos, ou em desuso, na cidade Universitária de Macaé-RJ e a execução de ações educativas e a troca de saberes entre as comunidades universitária e escolares de ensino médio de Macaé e região.

O sistema de logística reversa apresentado no presente trabalho foi implantado com sucesso, permitindo destinar corretamente $21,2 \mathrm{Kg}$ de resíduos de medicamentos durante o ano de 2019, o que representa 1001 unidades de diferentes formas farmacêuticas (sólidas, semissólidas e líquidas). Além disso, a caracterização dos materiais recebidos através do dispositivo contentor permitiu observar que $68,8 \%$ dos medicamentos apresentavam o prazo de validade expirado e que $24,4 \%$ foram descartados ainda com o prazo de validade vigente, sendo $78,7 \%$ dos medicamentos isentos de controle especial. Da mesma forma que o gerenciamento dos resíduos as atividades "Posso jogar remédio no lixo?" apresentadas no trabalho foram de grande sucesso, atingindo um ótimo nível de satisfação junto aos públicos participantes e evidenciado através dos formulários de avaliação externa.

O presente trabalho tem, portanto, contribuição significativa para a temática do descarte de medicamentos, demonstrando a importante atuação na redução do impacto ambiental através do destino adequado de resíduos potencialmente danosos, assim como através do estabelecimento de procedimentos para o gerenciamento dos processos do manejo desses materiais. O impacto social, por sua vez, foi obtido através da educação continuada e difusão do conhecimento junto aos públicos participantes, formando recursos humanos como potenciais transformadores da sociedade. Já o impacto na formação do estudante de graduação se dá pelo convívio com as demandas da sociedade, reflexão sobre o tema e seus impactos, interação com empresa parceira do projeto, bem como com público externo à universidade.

A interprofissionalidade e interdisciplinariedade aconteceu no desenvolvimento das 
atividades, uma vez que a interação com outros setores da sociedade trouxeram reflexões fundamentais sobre a melhoria contínua das práticas de gestão e educacionais relacionadas ao descarte de medicamentos provenientes de domicílios e de estabelecimentos comerciais e unidades de saúde, assim como a necessidade constante de ampliar a troca de saberes envolvendo o uso racional de medicamentos, automedicação e a atenção farmacêutica. É neste contexto que perspectivas importantes vêm sendo planejadas e executadas no âmbito do projeto, estando estas diretamente ligadas à aplicação de questionários para estudar as percepções e práticas relacionadas ao descarte de medicamentos adotadas pela comunidade e pelos estabelecimentos farmacêuticos, uma vez que estes últimos poderão ter necessidade de atualizações em práticas sinalizadas nas legislações recentemente publicadas. O projeto vem conduzindo, ainda, a elaboração de seu plano de gerenciamento de resíduos e sistema de procedimentos operacionais padrão, tendo como ambição a ampliação das ações educativas a diferentes públicos-alvo através de circuitos itinerantes com recebimento de medicamentos vencidos ou em desuso, além de treinamentos a organizações público-privadas, inclusive com a possibilidade do uso de ferramentas virtuais para ampliar o acesso à informação, diálogo e serviço à comunidade. Dessa forma, o trabalho contribui para a saúde pública e ambiental, gerando impacto na sociedade, transformação social e humana. Serve ainda como fonte de consulta para novos projetos e ações de extensão e pesquisa e para estimular iniciativas relacionadas ao tema.

\section{AGRADECIMENTOS}

Os autores agradecem o Programa Institucional de Fomento Único de Ações de Extensão (PROFAEX) da Universidade Federal do Rio de Janeiro, as escolas participantes das atividades educativas e a empresa Transforma Gerenciamento de Resíduos pela parceria no projeto de extensão.

\section{REFERÊNCIAS}

AGÊNCIA NACIONAL DE VIGILÂNCIA SANITÁRIA. O que devemos saber sobre medicamentos. Brasília: ANVISA, 2010. Disponível em: https://www.gov.br/anvisa/ptbr/centraisdeconteudo/publicacoes/medicamentos/publicacoes-sobre-medicamentos/o-quedevemos-saber-sobre-medicamentos.pdf/view. Acesso em: 08 abr. 2021. 
ASSOCIACAO BRASILEIRA DE NORMAS TECNICAS. NBR ISO 10015: Gestão da qualidade: Diretrizes para gestão da competência e desenvolvimento de pessoas.

Rio de Janeiro, 2020.

ASSOCIAÇÃO BRASILEIRA DE NORMA TÉCNICAS. NBR 16457: Logística reversa de medicamentos de uso humano vencidos e/ou em desuso - Procedimento. Rio de Janeiro, 2016.

ALMEIDA, L. A. Descarte domiciliar de medicamentos indesejáveis. Dissertação (Mestrado) - Programa de Pós-graduação em Saúde Coletivo do Centro de Ciências da Saúde da Universidade Estadual do Ceará. Fortaleza-CE, 80 p., 2017.

BALDONI, A. O.; GONTIJO, J. D.; SILVA, V. K. C.; FERNANDES, M. R.; ALVIM, C. P.; FERREIRA, S. M.; ANDRADE, R. C. M.; SOUSA, F. M.; RODRIGUES, L. A.; ARAÚJO, M. G. F.; SANCHES-GIRAUD, C.; DINIZ, R. S.; PEREIRA, M. L.; FIGUEIREDO, R. C. Armazenamento e descarte de medicamentos: estratégia educativa e perfil de medicamentos descartados. Extensio: Revista Eletrônica de Extensão, v. 12, n. 20, p. 48-61, 2015.

BILA, D. M., DEZOTTI, M. Fármacos no meio ambiente. Química nova, v. 26, n. 4, p. 523 530, 2003.

BRASIL. Ministério da Saúde. Portaria no 3.916, de 30 de outubro de 1998. Aprova a Política Nacional de Medicamentos. Diário Oficial da União, Brasília, DF, 30 de outubro de 1998.

BRASIL. Ministério da Saúde. Portaria nº 2.914, de 12 de dezembro de 2011. Dispõe sobre os procedimentos de controle e de vigilância da qualidade da água para consumo humano e seu padrão de portabilidade. Diário Oficial da União, Brasília, DF, 14 de dezembro de 2011.

BRASIL. Agência nacional de vigilância sanitária (ANVISA). Resolução de Diretoria Colegiada $\mathrm{n}^{\circ} 222$, de 28 de março de 2018. Dispõe sobre as boas práticas de gerenciamento dos resíduos de serviços de saúde. Diário Oficial da União 2019; 29 março de 2018.

BRASIL. Agência Nacional de Vigilância Sanitária (ANVISA). RDC no 20, de 5 de maio de 2011. Dispõe sobre o controle de medicamentos à base de substâncias classificadas como antimicrobianos, de uso sob prescrição, isoladas ou em associação. Diário Oficial da União, Brasília, DF, 9 de maio de 2011.

BRASIL. Conselho Nacional de Saúde (CNS). Resolução - RDC no 510, de 07 de abril de 2016. Dispõe sobre as normas aplicáveis a pesquisas em Ciências Humanas e Sociais. Diário Oficial da União, Brasília, DF, 24 de maio de 2016.

BRASIL. Conselho Nacional de Saúde (CNS). Resolução nº 466, de 12 de dezembro de 2012. Dispõe sobre diretrizes e normas regulamentadoras de pesquisas envolvendo seres humanos. Diário Oficial da União, Brasília, DF, 13 de junho de 2013.

BRASIL. Decreto $n^{\circ} 10.388$, de 5 de junho de 2020. Regulamenta o $\S 1^{\circ}$ do caput do art. 33 da Lei $\mathrm{n}^{\circ}$ 12.305, de 2 de agosto de 2010, e institui o sistema de logística reversa de medicamentos domiciliares vencidos ou em desuso, de uso humano, industrializados e 
manipulados, e de suas embalagens após o descarte pelos consumidores. Diário Oficial da União, Brasília, DF, 05 de junho de 2020.

BRASIL. Instituto Nacional de Estudos e Pesquisas Educacionais Anísio Teixeira. Plano Nacional de Educação PNE 2014-2024: Linha de Base. Brasília, DF: Inep, 2015.

BRASIL. Lei $\mathrm{n}^{\circ}$ 12.305, de 2 de agosto de 2010. Institui a Política Nacional de Resíduos Sólidos; altera a Lei no 9.605, de 12 de fevereiro de 1998; e dá outras providências. Diário Oficial da República Federativa do Brasil, Poder Executivo, Brasília, DF, 03 de agosto de 2010 .

BRASIL. Lei $\mathrm{n}^{\circ}$ 5.991, de 17 de dezembro de 1973. Dispõe sobre o Controle Sanitário do Comércio de Drogas, Medicamentos, Insumos Farmacêuticos e Correlatos, e dá outras Providências. Diário Oficial da República Federativa do Brasil, Poder Executivo, Brasília, DF, 19 de dezembro de 1973.

BRASIL. Portaria $\mathrm{n}^{\circ}$ 344, de 12 de maio de 1998. Aprova o Regulamento Técnico sobre substâncias e medicamentos sujeitos a controle especial. Diário Oficial da União, Brasília, DF, 15 de maio de 1998.

COUNCIL OF THE EUROPEAN COMMUNITIES. (2008) Directive on environmental quality standards in the field of water policy (directive 2008/105/EC). Official Journal of European Community, L 348, p. 84-97.

COUNCIL OF THE EUROPEAN COMMUNITIES. (2015) Commission implementing decision establishing a watch list of substances for Union-wide monitoring in the field of water policy (Commission implementing decision 2015/495). Official Journal of European Community, L 78, p. 40-42.

ROCHA, Bruno Simas da; HEINECK, Isabela; AMADOR, T. A.; SEIXAS, L. M. J.; GALliNA, S. M.; SALVALDORETI, C.; BORGES, P. E. M. Caracterização dos medicamentos descartados por usuários da farmácia popular do Brasil/ farmácia-escola da UFRGS. Trabalho apresentado no 9 Salão de Extensão Universitário da Universidade Federal do Rio Grande do Sul. Porto Alegre - RS, 2009.

LIMA, D. R. S.; TONUCCI, M. C.; LIBÂNIO, M., AQUINO, S. F. D. Fármacos e desreguladores endócrinos em águas brasileiras: ocorrência e técnicas de remoção. Engenharia Sanitária e Ambiental, v. 22, n. 6, p. 1043-1054, 2017.

MARINI, D. C.; OLIVEIRA, E.D.S. Farmácia caseira e o descarte de medicamentos de moradores da cidade de Itapira - SP. FOCO: Caderno de Estudos e Pesquisas, v. 9, 2015.

MENEZES, I.; PAULIMO, B.; BELIZÁRIO, G.; KOROYVA, P.; FREITAS, P.; RIBEIRO MISTURINI, F. D. Análise da relação entre o uso racional de medicamentos e o descarte de medicamentos vencidos. Trabalho de conclusão de curso. Farmácia. Universidade Federal do Rio Grande do Sul-RS. Porto Alegre-RS, 45 p., 2015. 
NETO, L. M. Determinação do perfil do descarte de medicamentos no âmbito do centro universitário São Camilo (CUSC), São Paulo: Projeto piloto. III Simpósio de Assistência Farmacêutica. Centro Universitário São Camilo. São Paulo-SP, 21-23 de maio, 2015.

NUNES, P. L.; DE MORAES, M. M. S.; BANDEIRA, V. A. C. Descarte de medicamentos em uma farmácia universitária. Trabalho apresentado no Salão do Conhecimento UNIJUÍ XIX Jornada de Extensão. Ijuí-RS, 01-04 de outubro de 2018.

PINTO, N. B., LUSTOSA, J. P. G., de Alustau Fernandes, M. D. C. O descarte incorreto de fármacos e seus impactos no meio ambiente e na saúde pública. Revista de Pesquisa Interdisciplinar, v. 2, n 2.0, 2019.

RAMOS, H. M. P.; CRUVINEL, V. R. N.; MEINERS, M. M. M. D. A.; QUEIROZ, C. A.; GALATO, D. A. Y. A. N. I. Descarte de medicamentos: uma reflexão sobre os possíveis riscos sanitários e ambientais. Ambiente \& Sociedade, v. 20, n. 4, 2017.

SINITOX (Sistema Nacional de Informações Tóxicos-Farmacológicas). Disponível em: https://sinitox.icict.fiocruz.br/dados-nacionais. Acesso em: 08 abr. 2021.

SOARES, M. N.; ARTICO, L. L.; MENEZES, A. P. S. Analysis of discarded drugs in a waste management system in the city of Bagé-RS/ Brazil. Revista da Jornada da Pós-Graduação e Pesquisa - Congrega, 2017.

WORLD HEALTH ORGANIZATION (WHO) - Collaborating Centre for Drug Statistics Methodology. Oslo, Norway: Guidelines for ATC classification and DDD assignment, 2021. 